$225-236$

\title{
"EL INFIERNO SON LOS OTROS": APROXIMACIONES A LA CUESTIÓN DEL OTRO EN SARTRE Y LEVINAS ${ }^{1}$
}

"Hell is other people": An approach to the question of the Other in Sartre and Levinas

Juan Carlos Aguirre García*

Resumen

En la obra dramática A puerta cerrada (1944), el filósofo y literato Jean-Paul Sartre plantea la relación interpersonal como plena de conflicto e imposibilidad para llevar a cabalidad los ideales dialógicos. Las conclusiones a las que llega Sartre serán confrontadas con la propuesta del filósofo judío Emmanuel Levinas, con el fin de vislumbrar sus tesis en torno a la alteridad, mostrando cómo, en vez de acuerdos racionales que superen lo diverso y lo engloben en la totalidad, habrá que recibir al Otro y responderle en la trascendencia que exige su infinitud.

Palabras clave: Infinito, infierno, otro, palabra.

Abstract

The Philosopher and Writer Jean-Paul Sartre, in the dramatic work No Exit (1944), claims the inter-subjective relationship is full of conflict and that it is impossible to reach the dialogical ideal. We will confront Sartre's conclusions from the proposal of the jewish philosopher Emmanuel Levinas in order to see his thesis about otherness. We will explain how, instead of some rational agreements that overcome the diversity inside totality, it is necessary to receive the other and respond to him in the transcendence that Infinity requires.

Key words: Infinity, hell, other, word.

\section{EL INFIERNO COMO IMPOSIBILIDAD DE COMUNICACIÓN}

Al hablar hoy de infierno podemos caer en el riesgo de enunciar una palabra sin referente. Para evitar confusiones, es necesario advertir que a lo largo del texto la palabra infierno estará vacía de cualquier referencia teológica a lo que se denomina novísimos. ${ }^{2}$ Como se advierte en el título, el artículo remite a Sartre, el filósofo y dramaturgo francés, tan polémico en su tiempo. Infierno tendrá que entenderse,

\footnotetext{
${ }^{1}$ Este texto hace parte de las discusiones adelantadas al interior del grupo de investigación Fenomenología y Ciencia, adscrito a la Vicerrectoría de Investigaciones de la Universidad del Cauca - Popayán (Colombia). El autor desea dar expreso crédito y mención a la Universidad del Cauca por los espacios y tiempos permitidos para la realización de estas reflexiones.

${ }^{2}$ Por novísimos se entienden: "1. mors (muerte temporal de cada individuo); 2. mortuorum resurrectio (la resurrección de los muertos); 3. extremum iudicium (el juicio final); 4. infernum s. mors aeterna (infierno o muerte eterna); 5. vita aeterna (vida eterna)" (Araya, 81).
} 
entonces, desde la frase que Sartre pone en boca del Garcin, uno de los personajes de su ya clásica obra teatral: A puerta cerrada, frase que impide cualquier confusión: "Así que esto es el infierno. Nunca lo hubiera creído... ¿Recordáis?: el azufre, la hoguera, la parrilla... ¡Ah! Qué broma. No hay necesidad de parrillas; el infierno son los otros" (Sartre, APC 186). ${ }^{3}$ Este apartado tendrá como objetivo seguir la pista a la imposibilidad de la comunicación en la relación intersubjetiva, tal y como Sartre lo sugiere en la mencionada obra.

Sartre parte de un individuo aislado, cómodo en su espacio, preocupado por lo que obstaculiza su bienestar. Tal individuo, viéndose expuesto a la urgencia del desplazamiento, termina por volver familiar lo que a primera vista se hace extraño: "Yo... pienso que a la larga uno ha de habituarse a los muebles" (Sartre, APC 99). Pese a todo, nunca hay una instalación plena, siempre se está insatisfecho con lo presente: “¿Y por qué me han quitado el cepillo de dientes?" (Sartre, APC 101). Siempre se reclama un plus de dignidad. El familiarizar lo extraño y reclamar lo que se considera más propio, parecen manifestar la primacía de un yo cognoscente que se esfuerza por tomar bajo control toda situación, incluso aquella que desborda todo su poder comprehensivo. Aun en el infierno, Garcin reprocha al camarero: "No gritaré, no gemiré, pero quiero mirar la situación de frente. No quiero que me salte encima por detrás, sin que pueda reconocerla" (Sartre, APC 103). La seguridad y autarquía provienen, entonces, de un cogito que homologa, a la medida de sus representaciones, todo lo exterior, tornándolo mismidad.

Pero tanto la comodidad del habitar como el dominio que brota del pensar encuentran un desafío radical ante la presencia del otro. En A puerta cerrada, Sartre retrata un encuentro que, a su vez, prefigura la mayoría de los encuentros: sin conocer nada de él, Inés pregunta vehementemente a Garcin: “¿Dónde está Florence?" (APC, 112). Y, ante su silencio, reitera la pregunta exigiendo respuesta. Garcin, antes que indolente, le replica: "No sé nada" (ibid.). Este encuentro es el de dos mundos: el de Inés y el de Garcin, cada uno con sus moradas y sus seres queridos u odiados. Inés ve a Garcin como quien está destinado a infligirle: "¿Ah, usted? Usted es el verdugo" (Sartre, Huis Clos 122); en efecto, en cuanto no familiar, es decir, diverso, se establece que el otro está ahí como enemigo.

También Estelle, quien llegó después a la habitación, vio en Garcin una imagen de terror; sin trabar palabra alguna se dirige a él y exclama: “¡No! No, no, no levante la cabeza. Sé lo que ocultas con las manos, sé que ya no tienes rostro" (Sartre, APC 117). Los encuentros narrados por Sartre dan cuenta de motivos psicológicos que impiden una relación neutral con el otro; cada cual ve en el otro lo que espera ver $y$, aun en la diferencia, continúan haciéndose

\footnotetext{
${ }^{3}$ Por lo regular se usará la traducción al castellano referenciada en la bibliografía. En contadas ocasiones, sobre todo cuando haya confusiones, se recurrirá a la versión francesa (Sartre, Huis Clos). La versión castellana será abreviada como APC.
} 
presentes, como proyecciones, las ideas afincadas en el yo. Pero más que psicológicos, son auténticos factores ontológicos los que impelen al sujeto a anclarse centrípetamente en su ipseidad, quedándose encerrado en sí mismo, incapaz de apertura plena.

La reacción frente al otro, la desconfianza que su presencia me genera, pero a la vez su recurrencia ineludible, su permanente estar ahí, exige adoptar maneras que contribuyan a un habitar pacífico

Comprendo muy bien que mi presencia la importune. Y personalmente preferiría quedarme solo; tengo que poner mi vida en orden y necesito concentrarme. Pero estoy seguro de que podremos adaptarnos el uno al otro: no hablo, no me muevo y hago poco ruido. Solo que, si puedo permitirme un consejo, tendremos que mantener entre nosotros una extremada cortesía. Será nuestra mejor defensa (Sartre, APC 114).

En este sentido, el otro se tolera en tanto yo pueda conservar la primacía de mi pensar y no traspase la frontera de mi interioridad.

Sin embargo, aunque racionalmente se establezcan acuerdos, los profundos fantasmas que brotan de los más profundos abismos de la conciencia se imponen: "Es lo que le reprocho (Tic de Garcin) ¡Otra vez! Presume de cortés y no controla su cara. No está usted solo y no tiene el derecho de infligirme el espectáculo de su miedo" (Sartre, APC 114-115). Ni los gestos involuntarios del otro, ni una reacción frente al sofoco, ni la palabra desnuda, ni la certeza de que se está ante el otro por puro azar, porque nos tocó compartir destino; nada de esto nos permite conservar los acuerdos racionales, estos motivos ocultos fortalecen la caparazón que endiosa la identidad $(A=A)$, nos recluyen en el silencio. Luego del intento vano de convivir cortésmente en la diferencia, y sus respectivas fricciones, exclama Garcin: "No seré su verdugo. No les deseo ningún mal y no tengo nada que ver con ustedes. Nada. Es sencillísimo, será así: cada uno en su rincón; es la farsa. Usted ahí, usted ahí y yo aquí. Y silencio. Ni una palabra; no es dificil..." (Sartre, APC 134).

Pero en el fondo, los tres personajes saben que sí lo es. Y por eso intentan romper la atmósfera de silencio que se prometieron cumplir y que creían que era fácil; por eso Inés recita un poema en voz alta y Estelle demanda un espejo para afirmar una existencia que no se basta en el palpar; por eso, una trivial excusa retorna la palabra, ahora la de dos mujeres que intentan hacer a un lado al hombre, lo totalmente diverso, el obstáculo más profundo para desnudarse de modo pleno. Sin embargo, vuelve el fantasma del miedo, la creencia en que ambas se harán daño; aunque, a fin de cuentas, el sufrimiento es la posibilidad más real en el contacto entre humanos. Pero el sufrimiento puede esconderse en deseo, en intento de posesión del otro; Inés considera que si hay que sufrir, lo mismo da que sea por Estelle; Inés se cree con el mismo gusto de Estelle, Estelle se sorprende e Inés concluye: "Tengo tu gusto, puesto que me gustas" (Sartre, APC 139). 
No es extraño en la cotidianidad que se suprima la diferencia en aras de lo que nos unifica; más aún, en aras de la unidad recurrimos a abstracciones que engloban, bajo lemas de amor, justicia, igualdad, fraternidad. Pero el pensamiento de lo mismo olvida que el otro es otro que no se siente satisfecho cuando tiene en frente otra mirada: "No sé. Usted me intimida. Mi imagen en los espejos estaba domesticada. La conocía tan bien... Voy a sonreír: mi sonrisa irá hasta el fondo de sus pupilas y sabe Dios en qué se convertirá" (Sartre, APC 140). En el rostro del otro, en su mirada, se comprende hasta los huesos que es distinto de mí, que encierra una soledad inexpugnable, un silencio que no puedo violentar, así lo seduzca, lo abrace, así me grite: soy tuya.

Reaparece el círculo vicioso: la desconfianza que se incoa en el contacto con el otro, el temor que impide una exposición plena en el lenguaje, el conflicto que brota del lenguaje-palabra o del lenguaje-gesto y, finalmente, la pretendida vuelta al silencio para no hacerse daño: "¡Pero cállense! (Una pausa) Nos sentaremos de nuevo tranquilamente, cerraremos los ojos y cada uno tratará de olvidar la presencia de los demás" (Sartre, APC 142-143). Sísifo creyendo que alcanzará su cima, personajes que buscarán un modo de tolerar la presencia del otro con nuevos intentos, explorando nuevos modos de enunciación, proponiendo nuevos reposos con la intención de pensar mejor y volver a empezar sin fricciones. Pero Sísifo abruptamente es retirado de su objetivo por fuerzas que él no puede controlar; sin embargo, su condena es volver a empezar. Igual suerte corren los personajes de Sartre, con la certeza, cada vez más angustiante, de que cada intento será vano y de que siempre el contacto con el otro degenerará en conflicto, aun cuando se adopte la defensa del silencio, pues, como lo reconoce Inés frente a Garcin,

Lo siento a usted hasta en los huesos. Su silencio me grita en las orejas. Puede coserse la boca, puede cortarse la lengua, ¿Eso le impedirá existir? ¿Detendrá su pensamiento? Lo oigo, hace tic tac, como un despertador y sé que usted oye el mío. Es inútil que se arrincone en su sofá, está usted en todas partes; los sonidos me llegan manchados porque usted los ha oído al pasar (Sartre, APC 143).

Al borde del paroxismo que genera el eterno devenir se revelan las más crudas verdades de las relaciones humanas: el intento por escapar de los otros, de todos, pero la constante certeza de que la puerta está cerrada por fuera; por tanto, la ineludible responsabilidad por el otro, pues "ninguno de nosotros puede salvarse solo; tenemos que perder juntos o salir juntos del apuro. Elija" (Sartre, APC 156); la elección por el salir juntos, pero la seguridad de que el otro me está tendiendo una trampa y el asco que esto genera, el asco que el otro me provoca. En consecuencia, el desespero:

\footnotetext{
¡Abran! ¡Abran, pues! Lo acepto todo: los borceguíes, el plomo derretido, las tenazas, el garrote, todo lo que quema, todo lo que desgarra; quiero sufrir de veras. Antes cien mordiscos, antes el látigo, el vitriolo, que este padecimiento mental, este fantasma del sufrimiento que roza, que acaricia y nunca hace bastante daño (Sartre, APC 179).
} 
Las relaciones que se han ido tejiendo conducen a la certeza de la presencia absoluta del otro; no estamos solos y juntos tenemos que hacernos más grato el momento; sin embargo, frente al otro siento profunda desconfianza, incapacidad de compasión, temor a que me haga sufrir. Tales sentimientos se exacerban, pues cada vez que actúo o me expongo en la palabra, voy quedando desnudo, voy mostrando quién soy y el otro jamás se mostrará plenamente. En la relación se exige la reciprocidad y nadie se atreve a dar el primer paso: "si quieres mi confianza tienes que empezar por entregarme la tuya" (Sartre, APC 176). El juego de las ideas culmina en que la sagacidad de la razón se convierte en la trampa que me impide el acceso al otro.

\section{DEL INFIERNO DE LA TOTALIDAD A LA INFINITUD DEL OTRO}

Se ha planteado en el anterior apartado que la relación interhumana está influenciada por múltiples factores que hacen de ella un fenómeno complejo. A partir de la obra de teatro escrita por Sartre esbozamos la conclusión que estamos juntos, inexorablemente juntos, y que esta certeza se nos exhibe como problemática. Quisiéramos circunscribir el estado de angustia existencial, infierno en la terminología sartreana, a una cuestión de lenguaje, en tanto es este el que nos comunica y aleja, el que intenta salir del estado deplorable a una esfera distinta. A partir del texto de Sartre hemos dejado la situación en su más radical aporía; sin embargo, este discurso, aunque inherente a lo humano, puede encontrar salidas. Este apartado recurrirá a algunas ideas del filósofo Emmanuel Levinas, las cuales apenas serán sugeridas con el fin de llevar a su extrema radicalidad la angustia sartreana, pero, a la vez, plantear la discusión en otro terreno, permitiéndonos una consideración alternativa y esperanzadora para el fenómeno dialógico.

Podemos ver a Sartre como un filósofo que, implicado en la literatura, denuncia el desgaste de la tradición occidental, una tradición que, contraria a los deseos del Iluminismo, culmina en guerra, y guerra que amenaza con la aniquilación definitiva. En Sartre se halla un diagnóstico de lo que Levinas denomina totalidad, concepto que domina la filosofía occidental, especialmente en su línea ontológica. Como características de la totalidad, Levinas señala, entre otras: a. la pérdida de la identidad: "los individuos son meros portadores de fuerzas que los dirigen a sus espaldas" (Levinas, TI 6); ${ }^{4}$ b. la postergación del presente en aras de una salvación prometida: "porque solo el último sentido cuenta, solo el último acto transforma los seres en sí mismos" (ibíd.); c. la paz brota mediante estrategias políticas de pacificación, dejando a los individuos alienados: "no devuelve a los seres alienados su identidad perdida" (ibíd.); y, d. la política se impone a la moral.

A esa ontología de la guerra, típica de la totalidad, Levinas opone, utilizando una terminología profética, lo que él denomina la Escatología de la paz mesiánica, la

\footnotetext{
${ }^{4} \mathrm{La}$ abreviatura TI corresponde a la obra de Levinas Totalité et infini.
} 
cual no consiste en exponerse con evidencia filosófica, como para hacerse visible en el contexto del pensamiento. Tal escatología no puede pensarse como un complemento a las evidencias filosóficas, como si pudieran cohabitar en un mismo espacio, pues "reducida a las evidencias, la escatología aceptaría desde el principio la ontología de la totalidad emergida de la guerra" (Levinas, TI 7). El alcance auténtico de la escatología es otro, a saber: ante la alienación del ser, pone en relación con el ser, más allá de la totalidad o la historia; tal relación implica una excedencia siempre exterior a la totalidad. Ahora bien, a diferencia del clásico sentido de escatología, Levinas no habla de estadios ultramundanos, evasiones de la realidad; el más allá "se refleja en el interior de la experiencia. La escatología, en tanto que el «más allá» de la historia arranca los seres a la jurisdicción de la historia y del porvenir, los interpela en su plena responsabilidad y a ella los convoca" (ibid.).

El espacio en el que transcurren los diálogos que narra Sartre da cuenta de un estado cerrado, de una totalidad sin posibilidad de un más allá, de una habitación cerrada por fuera. Cada uno de los tres personajes habla de sus mundos particulares, justifica sus historias, sus condenas, como si fueran el fruto de un transcurrir inevitable de la historia del cual no cabe culpa alguna. Los diálogos que se trenzan siempre apelan a las tácticas conversacionales, más que al desnudamiento. La escatología propuesta por Levinas como alternativa a la totalidad no exigiría la apertura de la puerta, la salida a otra habitación, a una habitación para cada uno de los condenados. En tanto "todas las causas están maduras para ser entendidas" (Levinas, TI 8), no se habla de un juicio al final de los tiempos, sino que se somete la historia a juicio, juicio de todos los momentos en el tiempo en que se juzga a los vivos, "la idea escatológica de juicio [...] implica que los seres tienen una identidad «antes» de la eternidad, antes de la consumación de la historia, antes de que los tiempos sean cumplidos, mientras que aún hay tiempo, implica que los seres existen en relación, pero a partir de sí y no a partir de la totalidad" (ibíd.). En este sentido, la escatología proporciona una paz distinta al infierno sartreano, un infierno que se torna injusto, como si se estuviera en él por error; el ser que desborda la historia, la totalidad, se asume como comprometido y personal, como capaz de hablar: "la paz se produce como una aptitud para la palabra" (ibid.).

Tenemos, entonces, que la solución no se halla inventando o prometiendo paraísos; si bien la única posibilidad no se encuentra en la totalidad, su ruptura no implica dirigirnos a otro lugar, pues a donde vayamos nos toparemos con el rostro interpelante del otro. La cuestión remite, más bien, a una exterioridad que no se contente con habitar los modos y costumbres impuestos por poderes ajenos, sin asumir la exigencia de responder. La exterioridad o trascendencia comienza a romper el cerco de la pieza cerrada de Sartre cuando, reconociendo la identidad del Mismo, nos topamos de frente con el rostro del otro, cuando el infinito se nos revela.

Podría decirse que quien primero llega al salón, aunque consciente del destino eterno que le corresponde, procura instalarse y "sabe" que, a la larga, podrá habituarse 
a la decoración y los ritmos que el espacio suscite; aunque extraña un elemento de aseo, intenta configurar el mundo a la medida de su pensamiento, de modo que todo vaya haciéndose familiar y nada aparezca como sorpresivo. Sin embargo, como lo advierte Levinas, reflexionando sobre la morada, "el papel privilegiado de una casa no consiste en ser el fin de la actividad humana, sino en ser condición y, en este sentido, el comienzo. El recogimiento necesario para que la naturaleza pueda ser representada y trabajada, para que se perfile solo como mundo, se realiza como casa" (Levinas, TI 162). Por tal motivo, la comodidad de la casa no es un estado perpetuo, exige la salida al trabajo, el recogimiento, pero, sobre todo, la acogida. ${ }^{5}$

En efecto, el primer auténtico encuentro interhumano narrado por Sartre, el encuentro entre Garcin e Inés, marca un punto de inflexión de las consideraciones individuales; sin embargo, la presencia del otro, pese a su diferencia, impele tanto a Garcin como a Inés a acomodarlo a sus esquemas mentales, a hacerlo familiar como familiar es un sillón, un timbre o una estatua; aunque en lo más profundo, en los tuétanos, se sepa que el otro es distinto de mí y encierra, como ya se ha dicho, una soledad inexpugnable. Vista desde la perspectiva escatológica levinasiana, Sartre llega a la presentación del otro como absolutamente otro, como infinito; sin embargo, el recibir al otro con la pretensión de volverlo familiar, de amoldarlo a mis esquemas conceptuales (y cada uno de los intentos de los personajes de A puerta cerrada tiende hacia este fin), es visto como violencia, pues se quiere tener experiencia de él, de adecuarlo a mis expectativas, a sabiendas de que el otro es absolutamente otro, es infinito. Es por esto que:

La relación con lo infinito no puede, ciertamente, expresarse en términos de experiencia, porque lo infinito desborda el pensamiento que lo piensa. En este desbordamiento se produce precisamente su infinición misma, de tal suerte que será necesario aludir a la relación con lo infinito de otro modo que en términos de experiencia objetiva. Pero si experiencia significa precisamente relación con lo absolutamente otro —es decir, con lo que siempre desborda el pensamiento - la relación con lo infinito lleva a cabo la experiencia por excelencia (Levinas, TI 10).

Se plantean entonces dos cuestiones: por un lado, la relación con el otro tiene que superar las simples categorías cognoscitivas que intentan reducir toda experiencia a algo conocido; por otro lado, Levinas denuncia que no puede reducirse toda experiencia a experiencia objetiva, toda vez que se encierra aquí una trampa: ya que el otro es absolutamente otro, su carácter de infinito no se deja adecuar al pensamiento que lo piensa; en ese sentido, la actitud propia de la experiencia auténtica tendrá que bastarse con establecer una relación con lo absolutamente otro, la que no solo será siempre incompleta a los encasillamientos en los que se trata de contener, sino que también exigirá otros elementos por fuera de la tematización.

\footnotetext{
${ }^{5}$ Una presentación de este importante concepto levinasiano se puede encontrar en Aguirre García.
} 
Basándose en lo anterior, podemos analizar lo sucedido en los encuentros que se establecieron entre los tres personajes de Sartre. Si bien A puerta cerrada es una obra literaria, no puede negarse que, como en toda auténtica obra literaria, abundan elementos metafísicos que le sirven de resorte. ${ }^{6}$ En el caso específico de Sartre tenemos que él comparte con Levinas la visión del otro como aquel que supera su carácter de imagen reducida a experiencia: "la percepción del Otro-como-objeto se refiere a un sistema coherente de representaciones, y este sistema no es mío. Esto significa que, en mi experiencia, el Otro no es un fenómeno que se relacione con mi experiencia sino que, en principio, él mismo se refiere a un fenómeno localizado por fuera de toda experiencia posible para mí" (Sartre, EN 266). ${ }^{7}$ Sin embargo, Sartre inicia un camino distinto al de Levinas en torno a la cuestión del rostro ${ }^{8}$

En primer lugar, Sartre reconoce que la apariencia del Otro es un fenómeno demasiado complejo para ser reducido al problema de la imagen en el espejo:

Si aprehendo la mirada, ceso de percibir los ojos; ellos están ahí, permanecen en el campo de mi percepción como presentaciones puras, pero no hago uso de ellos. Ellos están neutralizados, puestos fuera de juego; no son más el objeto de una tesis que perdura en el estado de "desconexión" en el que la palabra es puesta por una conciencia que practica la reducción fenomenológica prescrita por Husserl (Sartre, EN 297).

Podría decirse que Sartre, aunque reconozca la precariedad de la percepción, continúa situado en el campo de lo perceptual; en este sentido, sus tesis continúan remitiendo al ver, a la iluminación, a la Luz.

En segundo lugar, Sartre propone una salida a la ausencia del Otro: "Si el Otro es esta ausencia, si su ser es 'no-objetivo', entonces es solo a partir de una similar 'conversión y degradación' que puede llegar a ser un objeto presente a la mano para mi uso en el dominio de lo práctico" (Watson, 46). Aquí pareciera que Sartre queda preso de la unidad sintética de la conciencia, la que se plantea como imposible de franquear: si el Otro es absolutamente Otro, tendré que reconocer que sigue siendo un objeto para mí, aunque distinto. En este punto, Sartre recurre a las figuras duales ya clásicas en la fenomenología francesa: "el Otro es presencia-ausencia intramundana", un "trascendencia-trascendido", "visible-invisible", imago, en sentido de Barthes, una clase de estado absoluto de la carne no susceptible de alcanzar o relegar.

\footnotetext{
${ }^{6}$ Esta idea se hace evidente en filósofos, ver por ejemplo Merleau-Ponty, Sense et non sense, especialmente su ensayo titulado Le roman et la métaphysique; pero también en novelistas, véase en este aspecto a Kundera.

${ }^{7}$ Con la abreviatura EN se referencia la obra de Sartre: L'être et le néant (1943).

${ }^{8}$ La presentación de Sartre se puede encontrar suficientemente esbozada en Watson. Sigo muy de cerca su análisis.

${ }^{9}$ Un exponente preclaro de las mismas es Merleau-Ponty, Le visible et l'invisible: suivi de Notes de travail.
} 
Los movimientos de conversión y degradación significan en Sartre que, una vez se aclare que la mirada del Otro se puede trascender, si el Otro es a fin de cuentas una "trascendencia-trascendida", entonces la diferencia entre el yo y el Otro, así como la diferencia entre el percepto y la imagen, se convierte también en una diferencia trascendida, llega a ser mío el trascender de esta trascendencia. Y, en el momento en el que el Otro es "objetivado", así también la verdad se desplaza. El mismo movimiento que restaura la dignidad en la distancia con el Otro, hace del rostro del Otro el detentador de la verdad. Pero precisamente en la destrucción de la objetividad y la totalización de mi mirada, y consiguientemente, en la destrucción del estricto subjetivar de la verdad, se da fuerza a una verdad que trasciende la mirada individual del por sí mismo (Watson, 47ss).

Si bien los análisis sartreanos del rostro exigen un desarrollo más profundo, que superarían las pretensiones de este escrito, baste decir con Levinas que "El rostro del otro destruye en todo momento y desborda la imagen plástica que él me deja" (Levinas, TI 43); en este sentido, la fenomenología del rostro, si es que puede hablarse de algo así en Levinas, ${ }^{10}$ se distancia de los análisis de Sartre en tanto estos permanecen en la consideración del Rostro del Otro como un Rostro que aunque muestra y oculta, sigue estando ahí, a disposición de quien lo observa, tornándose una clase de objeto. ${ }^{11}$

Las implicaciones que esto tiene para la relación Yo-Otro son muy relevantes: en primer lugar, a diferencia del Otro que aparece como verdugo en Sartre, ${ }^{12}$ en Levinas el Otro cuya epifanía acaece en el Rostro es un Infinito que supera toda representación que me haga de él; más aún, puedo recibirlo sin siquiera saber el color de sus ojos. En segundo lugar, el Rostro del Otro no me deja tranquilo, rompe el gozo de mi ipseidad, pero tampoco tengo como opción la indiferencia, el recurrir a la cortesía: el Otro siempre me exige una respuesta. En tercer lugar, en el encuentro con el Otro nunca aparece la posibilidad del ser verdugo del Otro, del Otro siempre se es rehén y el Otro se

\footnotetext{
${ }^{10}$ En entrevista con Phillipe Nemo, Levinas corrige a este cuando le pregunta en qué consiste y para qué sirve una fenomenología del rostro. Ante lo cual él responde que "no se puede hablar de una fenomenología del rostro en tanto la fenomenología describe lo que aparece; por lo mismo, tampoco se podría hablar de una mirada vuelta hacia el rostro, pues la mirada es conocimiento y percepción" (Levinas, EI). La abreviatura EI corresponde a la obra Ética e infinito.

${ }^{11}$ Para una mayor ampliación de la cuestión del Rostro en Levinas y su relación con el otro, ver Jaramillo y Aguirre, Rostro y alteridad.

${ }^{12}$ La adscripción del otro como verdugo es recurrente en la reflexión que confronta las posiciones de Sartre y Levinas. Por ejemplo, Shankman afirma: "Para Sartre, el Otro es una amenaza a la integridad del yo. Para Levinas, sin embargo, el Otro es lo que me saca de mi mundo encapsulado y soy el único responsable por su singularidad irremplazable" (56). Igualmente, Zahavi plantea que "El propio Sartre tomó la intersubjetividad como, primordialmente, una cuestión de conflicto y confrontación más que una coexistencia pacífica" (169). Sin embargo, Zahavi coincide en señalar que tanto Sartre como Levinas (aunque realmente la tesis de Zahavi es que este planteamiento ya estaba en Husserl) pusieron su énfasis en "la trascendencia del otro" (172); es decir, en este aspecto habría posibilidad de comparar ambos autores. En un texto más reciente, llamado Intersubjetividad, Zahavi plantea que "tanto para Sartre como para Levinas una explicación de la intersubjetividad fracasará si trata de eliminar la diferencia entre el yo y el otro".
} 
recibe en la pasividad: "Tal pasividad sin duda es una exposición del sujeto al otro, pero la pasividad del sujeto es más pasiva que la que sufre el oprimido condenado a la lucha. La pasividad más pasiva, la inasumible subjetividad o incluso la sujeción del sujeto dice relación con mi obsesión por la responsabilidad para con el oprimido otro que yo" (Levinas, AE 92). ${ }^{13}$ Finalmente, la palabra dirigida al Otro no tiene como fin persuadirlo, ni seducirlo; en la propuesta ética levinasiana la palabra se torna centro y acceso privilegiado al Otro; de nuevo suena el ya proclamado lema: "la paz se produce como esta aptitud para la palabra" (Levinas, TI 8). ${ }^{14}$

En tanto se había afirmado que la aporía sartreana conduce a una imposibilidad para el diálogo, podremos ver que, sin embargo, en Levinas se halla una alternativa a tal asunto; es por ello que este apartado concluirá con una reflexión en torno a la palabra. Aunque una elucidación de la filosofía del lenguaje en Levinas y sus posibilidades hermenéuticas requiere un ejercicio muy juicioso de análisis, podríamos condensar buena parte de sus intuiciones al respecto en la siguiente cita:

El lenguaje lleva a cabo, en efecto, una relación de tal suerte que los términos no son limítrofes en esta relación, que el Otro, a pesar de la relación con el Mismo, sigue siendo trascendente al Mismo. La relación del Mismo y del Otro - o Metafísica - funciona originalmente como discurso, en el que el Mismo, resumido en su ipseidad de 'yo' — de ente particular único y autóctono- sale de sí (Levinas, TI 28-29).

Es muy importante resaltar el aspecto del discurso, pues no está apelándose a recorridos místicos o a elucubraciones teológicas para el acceso al otro; si bien el lenguaje ha estado siempre de lado de la razón -incluso hubo una época en la que solo lo constatable en hechos era digno de enunciarse y de lo demás había que callarlo- es el lenguaje, obviamente no el dependiente exclusivamente de los hechos, el que permite preservar la alteridad. Como primeras insinuaciones respecto de este lenguaje, Levinas sostiene que en vez de funcionar como una operación sinóptica, el acercamiento mediante el lenguaje se da en la relación cara-a-cara; en tal sentido, la relación Mismo-Otro no anula al yo o al pensamiento, los exige: "El 'pensamiento', la 'interioridad' son la ruptura misma del saber y la producción (no el reflejo) de la trascendencia. No conocemos esta relación — por eso mismo notable — más que en la medida en que la efectuamos. La alteridad solo es posible a partir del Yo" (Levinas, TI 29). El yo no renuncia a su identidad y a su pensamiento, el yo hace uso del lenguaje; sin embargo, por estar inmerso en el discurso, reconoce al Otro un derecho sobre ese egoísmo y, en consecuencia, permite al otro justificarse: "la apología en la que el Yo a

\footnotetext{
${ }^{13} \mathrm{La}$ abreviatura AE corresponde a la obra de Levinas: Autrement qu'être ou au-dela de l'essence.

${ }^{14}$ Un primer intento de reconstrucción del asunto del lenguaje en Levinas puede encontrarse en Jaramillo Echeverri y Aguirre García, sobre todo el apartado 4: "Levinas y las ciencias sociales: entre la retórica de la injusticia y la instauración de la significación" (Jaramillo y Aguirre, Levinas y las ciencias sociales, 12-14).
} 
la vez se afirma y se inclina ante lo trascendente, está en la esencia del discurso" (ibid.).

En este punto encontramos un elemento valioso a la hora de comprender el infierno compartido por Garcin, Inés y Estelle; la justificación de tal estado de incomunicación radicaría en la pretensión de tomarlo todo bajo el control del pensamiento, de un pensamiento de lo Mismo en el que, pese al encuentro de rostros que aparecen, cada uno conserva su egoísmo en un intento fallido de apertura.

\section{CONCLUSIÓN}

La obra de teatro A puerta cerrada sirvió como pretexto en este artículo para considerar las tesis de dos fenomenólogos franceses, Jean-Paul Sartre y Emmanuel Levinas, en torno a la cuestión del Otro o intersubjetividad. Los análisis descarnados de Sartre concluyeron en la trascendencia del otro, pero además, negativamente, en la posibilidad de acceder a él de modo asertivo. Por su parte, Levinas, también señalando la trascendencia del otro mediante la categoría de Infinito, sugiere una ruta de posibilidad al horizonte desesperanzador en el que Sartre deja la intersubjetividad: la palabra. Si bien el camino aún tiene muchos intrincados obstáculos por superar, el análisis permite entrever que si bien la condición humana pareciera recluirnos en nuestros propios límites monádicos, al fin y al cabo hay una salida posible: la salida de sí mismo propiciada por el otro.

Universidad del Cauca* Departamento de Filosofía Claustro El Carmen, Calle $4 N^{\circ} 3-56$

Popayán-Cauca (Colombia) jcaguirre@unicauca.edu.co 


\section{OBRAS CITADAS}

Aguirre García, Juan Carlos. "El concepto 'morada' como categoría posible para la fundamentación de una ética ambiental". Luna Azul 25, 2007:110.

Araya, E. "De las cosas últimas, De novissimis, desde la perspectiva evangélica", Teología y vida 41.1. 2000:81-89.

Jaramillo, Luis Guillermo; Juan Carlos Aguirre. "Levinas y las ciencias sociales: Fundamentos epistémicos desde la alteridad”. Folios 31. 2010:3-20.

------- "Rostro y alteridad: de la presencia plástica a la desnudez ética", en Revista Latinoamericana de Ciencias Sociales, Niñez y Juventud 8.1. 2010:175-188.

Kundera, Milan. El arte de la novela. Barcelona: TusQuest, 2009.

Levinas, Emmanuel. Autrement qu'être ou au-dela de l'essence. Paris: Le Livre de Poche, 2004.

----- Ética e infinito. Madrid: Visor, 1991.

------ Totalité et infini: Essai sur l'extériorité. Paris: Le Livre de Poche, 1990.

Merleau-Ponty, Maurice. Le visible et l'invisible: suivi de Notes de travail. Paris: Gallimard, 1964.

------ Sense et non sense. Paris: Gallimard, 1996.

Sartre, Jean-Paul. "Huis Clos". Sartre, Jean-Paul. Théatre. 65. Paris: Gallimard, 1947:110-168.

------ La puta respetuosa / A puerta cerrada. Trad. Aurora Bernández. Bogotá: Orbis, 1983.

------ L'être et le néant: Essai d'ontologie phénoménologique. Paris: Gallimard, 1943.

Shankman, Steven. Other Otherness: Levinas, Literature, Transcultural Studies. New York: State University of New York Press, 2010.

Watson, S. "Reason and the Face of the Other". Journal of the American Academy of Religion 54.1. 1986:33-57.

Zahavi, Dan. "Intersubjectivity". Luft, Sebastian y Soren Overgaard. Routledge Companion to Phenomenology. London: Routledge, 2011:180-189.

------ Subjectivity and Selfhood: Investigating the First-Person Perspective. London: The MIT Press, 2005. 